\title{
Dutch universities and research laboratories set for major cuts
}

[PARIS] The Dutch government is about to announce deep cuts across its scientific research organizations and universities. The proposal has already been accepted in principle by the Liberal and Labour parties that make up the new coalition government.

The Liberal/Labour and Democrat alliance that has ruled the country for the past four years re-emerged victorious in the general election on 6 May, with the Labour partyled by prime minister Wim Kok polling 29 per cent and the Liberals 25 per cent. After two months of negotiations, the shape of the new ruling coalition and its programme is finally beginning to emerge.

An announcement of the cuts - which are estimated to be around 4 per cent before inflation annually until 2002 — is expected to follow the appointment of a replacement for the current science minister, Jo Ritzen.

The cuts will hit universities and research organizations hard. Their budgets are already stretched because of a recent decline in total research spending, which fell from 2.3 per cent of gross domestic product in 1987 to 1.94 per cent in 1997.

"It is disappointing that, whereas the new government's programmes frequently mention knowledge and technology, additional support has not materialized," complains one ministry official. "Recent cuts have already meant that there is no money to finance some projects needed to bring laboratories up to international levels."

Government spending on research this year totals about DFl5.5 billion (US $\$ 2.7$ billion), with about DFl3.45 billion of this coming from the Ministry of Education, Culture and Science. The ministry has a budget of DFl38.9 billion, with DFl21.7 billion spent on schools and DFl7.9 billion on higher education. The universities receive DFl4 billion, and spend DFl 2.4 million on research.

According to the new government's programme, the ministry's overall budget would increase by DFl0.8 billion a year to 2002. But the extra money will be spent mainly on reducing the size of classes in primary schools. There will also be a substantial increase in funding for information technology use in schools.

Research has fallen victim to the government's determination to curb public expenditure to reduce the budget deficit and to cut taxes. The proposed cuts would have an impact on almost every area of science spending. Universities will suffer cuts of around 3 per cent annually, with more than half the cuts (DFl75 million) falling on their research funding.
The science ministry's DFl1.3 billion budget for the country's research organizations, which include bodies such as the national basic research agency, the NWO, will be reduced by around 4 per cent (DFl45 million) a year.

One result will be increasing pressure on the Netherlands' contributions to international 'big science' projects. These currently account for around 10 per cent of the budget of the research organizations.

The budgetary decisions indicate a significant shift in the government's research efforts away from universities and research agencies towards specific state-led projects geared to socioeconomic goals. The new government programme includes plans to spend on such goals a sum almost equivalent to the proposed cuts in research. These new projects have been drafted by an Interdepartmental Committee for Economic Structure, dominated by the Ministry of Economic Affairs.

Apart from the boost for information technology in schools, projects earmarked for massive investment include the 'Watergraafsmeer' project to create a life and information sciences centre and business park at the University of Amsterdam; the 'Delft Cluster project' to boost research in civil engineering and transport at engineering institutes in Delft, Delft University of Technology and at TNO, the national applied research organization; and the 'Gigaport project' aimed at building a sophisticated information technology infrastructure.

A similar philosophy has guided the creation of a 'superleague' scheme, under which up to ten research schools will be chosen from the 100 or so centres of international excellence. They will be given half the DFl200 million annual funding earmarked in university budgets for extra support for the research schools.

The research schools were set up five years ago to sharpen the focus of research at the 13 research universities, by regrouping teams in particular disciplines. The reforms were aimed at concentrating resources on the best groups, increasing competition, and boosting the visibility of the country's research abroad. But these goals have been criticized by some researchers who argue that selection is better carried out at the level of individual groups (see Nature 385, 758; 1997).

The government this month selected the first six centres of excellence, each combining departments from several universities. These will receive a total of DFl245 million over the next five years. The winning schools are in astrophysics, photonics, biomedical genetics, materials, Earth sciences and the chemistry of catalytic converters.

Some are concerned that the cuts could be counterproductive. According to one ministry official, their scale is likely to hamper plans by the government to radically reform the research system.

Under these plans, approved by parliament last April, the NWO would be transformed from a research agency with a dozen of its own institutes into a research council restricted to setting research strategy, and taking over from universities responsibility for distributing up to 25 per cent of grants to university research groups (see Nature 390, 9; 1997). The NWO's institutes, such as the agency of Fundamental Research on Matter, would be merged with institutes of the Royal Netherlands Academy of Science to form a new body under the auspices of the academy.

DeclanButler

\section{Hubble homes in on the birth of bright stars}

[LONDON] The Hubble Space Telescope has been used to record a group of 50 young, ultra-bright stars 200,000 light years away in the Small Magellanic Cloud (SMC). They are believed to be the youngest massive stars ever seen in the SMC. Each star is around 300,000 times brighter than the Sun.

The discovery (right) will sharpen the picture astronomers have of how stars formed in distant galaxies after the Big Bang, says Mohammad Heydari-Malayeri of the Paris Observatory, who heads the team of astronomers that made the discovery. The SMC stars, like those before them, are made up mostly of hydrogen and helium.

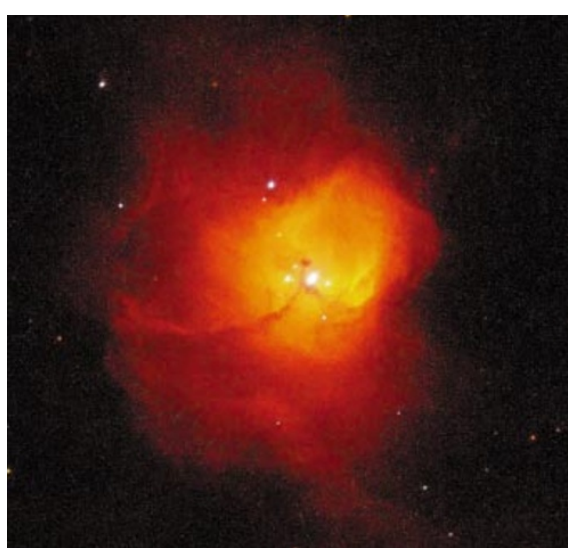

